\title{
Pollution des eaux dans le bassin du nakambe: cas du barrage de Ziga
}

\author{
Yacouba OUATTARA ${ }^{1}$, Issaka GUIGUEMDE ${ }^{1,2 *}$, Françoise DIENDERE ${ }^{2}$, \\ Jean DIARRA ${ }^{3}$ et Abdouraman BARY ${ }^{2}$ \\ ${ }^{1}$ Centre d'Etude pour la Promotion, l'Aménagement et la Protection de l'Environnement (CEPAPE) 03 \\ BP 7021 Université de Ouagadougou, Burkina Faso. \\ ${ }^{2}$ Laboratoire de Chimie Analytique, de Radiochimie et d'Electrochimie (LACARE), UFR/SEA ; \\ 03 BP 7021 Université de Ouagadougou, Burkina Faso. \\ ${ }^{3}$ Service Qualité- Eau de l'Office National de l'Eau et de l'Assainissement (ONEA) \\ 01 BP 170 Ouagadougou 01, Burkina Faso. \\ *Auteur correspondant, E-mail: issakguig@yahoo.fr; Tél. +22676651388.
}

\section{RESUME}

Le Burkina Faso est drainé essentiellement par quatre (4) grands bassins-versants: le bassin du fleuve Niger, le bassin du fleuve Nakanbé, le bassin du Mouhoun et le bassin de la Comoé. Le Nakanbé est le bassin le plus peuplé. La ville de Ouagadougou est presque entièrement tributaire des eaux de surface de ce bassin. Les problèmes d'approvisionnement en eau potable de la ville ont conduit à l'adoption du projet de barrage de Ziga. D'une capacité nominale de deux cents (200) millions de $\mathrm{m}^{3}$ au plan d'eau normal (P.E.N), cet important ouvrage a pour vocation l'approvisionnement en eau de la ville de Ouagadougou. Les populations riveraines du barrage pratiquent le maraîchage sur les rives et avancent dans le lit du barrage au fur et à mesure que l'eau se retire. Pour lutter contre les insectes et les animaux nuisibles (vers, rats, rats-voleurs, lièvres, perdrix, pintades sauvages, ...) qui détruisent leurs légumes, ils emploient des pesticides de synthèse, dont beaucoup sont interdits d'usage. Des fertilisants sont également utilisés. L'objectif de cette étude est de déterminer les perturbations physiques et chimiques subies par l'eau du barrage de Ziga par la mesure des paramètres physicochimiques de l'eau et la détermination des concentrations des résidus de pesticides organochlorés. Douze points de prélèvement d'échantillons ont été identifiés et ceux-ci ont été prélevés au mois de février et de mars 2012 et analysés pendant la même période. L'étude fait apparaître que les perturbations physicochimiques restent limitées et que sur les vingt (20) pesticides organochlorés recherchés, cinq (5) ont été détectés dans les eaux brutes du barrage de Ziga. Il s'agit de l'aldrine, de l'endosulfan sulfate, du alpha endosulfan, du beta $\mathrm{HCH}$ et du o,p- DDE.

(C) 2012 International Formulae Group. All rights reserved.

Mots clés: approvisionnement; eau; maraîchage; pesticides; paramètres physicochimiques.

\section{INTRODUCTION}

L'eau est une ressource indispensable à la vie humaine, mais y avoir accès n'est pas à la portée de tous (Batenbaum, 2012).

Toutes les grandes civilisations antiques sont nées grâce à la maîtrise de l'eau, sur les bassins de grands fleuves : Nil, Indus,
Tigre, Euphrate, Mékong, etc. (Cambon, 2008). Pendant longtemps, aller chercher de l'eau à la rivière, au puits ou à la fontaine fut une occupation quotidienne des populations. Avec l'essor des villes et des villages, les hommes ont inventé les canalisations pour rapprocher l'eau des zones d'habitation. C'est 
dans la seconde moitié du XIXème siècle que naissent les premières sociétés de distribution d'eau. La qualité sanitaire de l'eau potable devient alors un objectif majeur (Cambon, 2008). Bien que les années 1980 aient été baptisées «décennie de l'eau potable et de l'assainissement» par les Nations Unies, l'évolution générale de l'approvisionnement en eau par les populations n'incite guère à l'optimisme. En 2003, année internationale de l'eau douce, Kofi Annan, Secrétaire Général de l'ONU déclarait : " aucune mesure ne serait plus efficace pour combattre la maladie et pour sauver des vies dans le monde en voie de développement, qu'apporter une eau sûre et une hygiène adaptée à tous » (Djellouli et al., 2005). En effet, un milliard trois cents millions (1 300000 000) de personnes ne disposent pas toujours d'eau potable et les équipements d'assainissement font défaut à 1,7 milliard d'individus (OMS/UNICEF, 2007).

Dans le cas du Burkina Faso, pays enclavé en voie de développement, le seul service de distribution d'eau potable est l'Office National de l'Eau et de l'Assainissement (ONEA). Plus de quatre (4) millions d'habitants de la population du pays vivent dans le bassin du Nakanbé, qui est un des cours d'eau les plus importants du pays. Il prend naissance au Nord de Titao, sur un plateau de faible altitude et traverse le pays pour ensuite pénétrer au Ghana, où il alimente le réservoir du grand barrage d'Akosombo. Au site de Bagré, à environ $120 \mathrm{~km}$ en aval de Ziga, la rivière Nakambé a fait l'objet d'un aménagement ayant comme finalité l'irrigation de 7.400 ha et la production d'électricité. Dans la région de Ziga, la rivière Nakanbé s'écoule entre les mois de mai et janvier, le lit restant à sec entre février et avril de chaque année (Nacro, 2010).

La population de la ville de Ouagadougou qui était de 600000 habitants en 1995, contre 441500 habitants en 1985, est aujourd'hui estimée à environ un million huit cents mille (1 800 000) habitants, ce qui correspond à un taux de croissance annuel moyen d'environ 6,4\% (RGPH, 2006). Cette croissance galopante de la population n'est pas sans effet sur l'accroissement de la ville, créant d'autres problèmes, notamment celui de l'approvisionnement en eau potable. Le barrage de Ziga est prévu pour approvisionner en eau potable la ville de Ouagadougou au moins jusqu'en 2025. Celleci en effet, est presque entièrement tributaire des eaux de surface de ce barrage (MAHRH, 2001); il en est de même pour une grande proportion des populations se trouvant non loin du barrage, qui s'y rendent pour prélever l'eau destinée à la consommation et aux activités domestiques. Le bassin du barrage de Ziga (Figure 1), siège de nombreuses activités humaines, est confronté à divers types de pollutions, surtout avec les rejets de substances toxiques ou fertilisantes. Si un traitement chimique est appliqué à l'eau pour la débarrasser de ses substances bactériologiques, il n'en est pas de même pour les substances chimiques telles que les pesticides. Pourtant, la multitude des produits chimiques utilisés dans l'agriculture entraînent la pollution des sols, des eaux souterraines et de surface. Ainsi, on note la persistance de certaines pratiques dont la finalité est l'augmentation de la production: il s'agit de l'extension des superficies cultivées sur les berges des barrages et de l'utilisation accrue des engrais chimiques et pesticides. Quoique importante pour une croissance économique, ces pratiques n'intègrent pas toujours l'esprit de durabilité.

C'est dans cette optique que nous nous sommes intéressés à faire une étude sur la pollution des eaux dans le bassin versant du Nakanbé dont l'objectif principal est de rechercher les caractéristiques physiques et chimiques de l'eau de la retenue du barrage de Ziga pour déterminer l'état de pollution de cette eau car l'échéance de l'approvisionnement en eau potable de la ville de Ouagadougou au moins jusqu'en 2025 à partir de ce barrage ne pourra être atteinte que si la qualité de l'eau est préservée. 


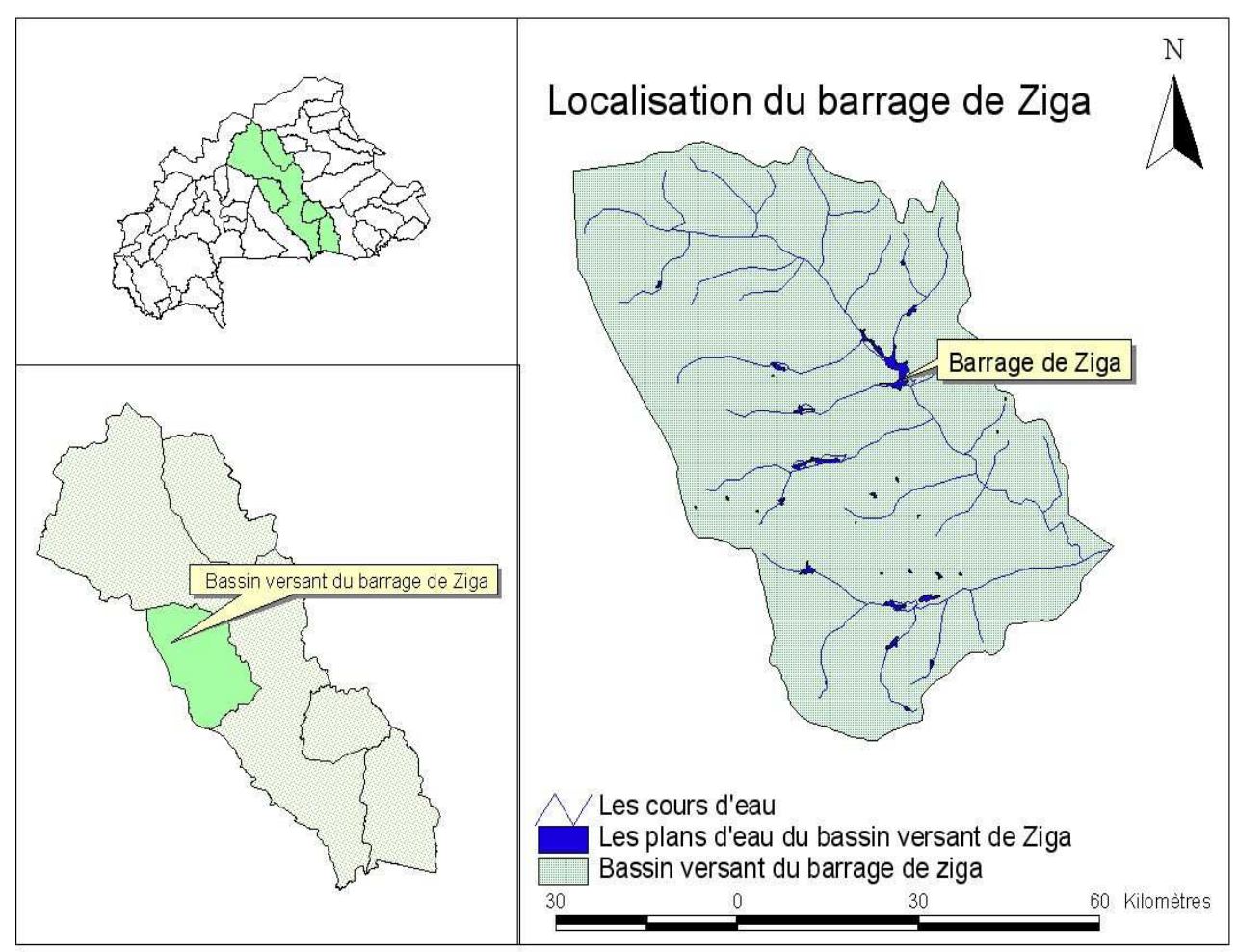

Figure 1 : Localisation du barrage de Ziga.

\section{MATERIEL ET METHODES}

\section{Collecte des données}

Des entretiens avec les maraîchers ont permis de connaître les différents pesticides utilisés sur les berges du barrage. Des visites sur le site ont permis d'identifier les points de prélèvement.

\section{Echantillonnage}

Douze points de prélèvements ont été identifiés. Leurs codes d'identification (levées GPS) et leurs positions sont présentés respectivement dans le Tableau 1 et sur la cartographie de la Figure 2. Ils sont situés en amont et en aval des cultures maraîchères, au niveau de la prise d'eau, en aval de la digue du barrage et comprennent aussi les points servant d'eau de boisson des populations autour du barrage. Les échantillons d'eau ont été prélevés selon les normes ISO 5667-1 et ISO 5667-2; ils ont été refroidis à une température voisine de $4{ }^{\circ} \mathrm{C}$ et conservés avec $10 \mathrm{ml}$ d'acide sulfurique $(4 \mathrm{~mol} / \mathrm{L})$. Les prélèvements ont été effectués aux mois de février et mars 2012 et à chaque fois, deux échantillons d'eau étaient mis dans deux flacons différents :

- une bouteille d'un litre en verre pour la détermination des concentrations des résidus des pesticides ;

-un flacon d'un litre en polyéthylène pour la détermination des paramètres physicochimiques. 


\section{Instrumentation et méthodologie}

Méthodes d'analyses des paramètres physico-chimiques

Nous avons résumé dans le Tableau 2 les méthodes et les appareils utilisés pour l'analyse des paramètres physico-chimiques.

\section{Méthodes de détermination des} concentrations des pesticides

L'appareil utilisé pour l'analyse des pesticides est un chromatographe en phase gazeuse (CPG) «Agilent 7890A » sans échantillonneur automatique ; il est muni d'un ordinateur avec un système incorporé pour le traitement des données (ChemStation). L'appareil permet le dosage des pesticides organochlorés dans les eaux potables, les eaux de surface, les eaux souterraines et les eaux usées. Sa limite de détection est de 0,005 $\mu \mathrm{g} / \mathrm{L}$ en teneurs individuelles en pesticides.

\section{Paramètres analysés}

Les paramètres physico-chimiques Les paramètres physicochimiques analysés sont les suivants :

La température, le $\mathrm{pH}$, la conductivité, les matières en suspension, la turbidité, les nitrites, les nitrates, les ions ammoniums, les orthophosphates et la chlorophylle « a ».

\section{Les paramètres chimiques}

Les paramètres chimiques analysés sont les organochlorés suivants :

Alpha-hexachlorocyclohexane (alpha$\mathrm{HCH}$; gamma-hexachlorocyclohexane (gamma-HCH); aldrine; o,p-DDE; dieldrine; o,p-DDD; beta-endosulfan; isodrine; endosulfan sulphate; o,p-DDT; beta-HCH; heptachlore; isobenzène; alpha endosulfan; $p$, p'-DDE; endrine; p,p'-DDD; méthoxychlore ; hexachlorobenzène (HCB) ; p,p'-DDT).

\section{Protocole expérimental de l'analyse des pesticides}

Le principe est mentionné dans la Norme européenne NF EN ISO 6468 de février 1997 remplaçant la norme expérimentale NF T 90120 d'avril 1985. Le principe est basé sur une extraction liquide-liquide des pesticides organochlorés par un solvant organique (hexane ou cyclohexane).

Toute la verrerie utilisée pour cette méthode (sauf la verrerie volumétrique) a été mise à l'étuve et chauffée à $450{ }^{\circ} \mathrm{C}$ pendant $2 \mathrm{~h}$ pour détruire la matière organique. La verrerie volumétrique a été rincée avec 2 à 3 portions d'hexane après lavage pour éviter les contaminations.

\section{Principe de fonctionnement du CPG}

Le principe repose sur les équilibres de concentration des composés présents entre deux phases non miscibles dont l'une dite stationnaire est emprisonnée dans une colonne et l'autre dite, mobile, se déplace au contact de la première. L'entraînement, à des vitesses différentes des composés présents par la phase mobile, conduit à leur séparation. A l'instant initial, le mélange à séparer est injecté à l'entrée de la colonne où il se dilue dans la phase mobile qui l'entraîne à travers la colonne. Les constituants du mélange, appelés généralement les solutés, sont inégalement retenus lors de la traversée de la colonne. Ils sont ainsi élués de la colonne les uns après les autres et donc séparés. Un détecteur placé à la sortie de la colonne, couplé à un intégrateur, permet d'obtenir le chromatogramme. Dans des conditions données, le "temps de rétention" caractérise qualitativement une substance. L'amplitude des pics, ou encore l'aire limitée par ces pics et la prolongation de la ligne de base permettent de mesurer la concentration de chaque soluté dans le mélange injecté.

\section{Manipulation}

La méthode d'analyse que nous avons utilisée est une méthode d'analyse multi résidus de détermination par chromatographie 
en phase gazeuse de résidus de pesticides. A chaque série d'analyse, nous passons un échantillon blanc (1 litre d'eau distillée acidifiée) et un échantillon de contrôle de concentration $0,1 \mu \mathrm{g} / \mathrm{l}$.

\section{Extraction}

L'extraction est réalisée sur 1 litre d'échantillon; on y ajoute $2 \mathrm{ml}$ de standard interne hexa bromobenzène (HBB à $200 \mu \mathrm{g} / \mathrm{l}$ ) et $30 \mathrm{ml}$ d'hexane puis l'on agite pendant 30 min à l'aide d'un agitateur magnétique. On transvase ensuite le contenu de la bouteille dans une ampoule à décanter et on laisse reposer jusqu'à ce que les deux phases soient bien séparées. On recueille l'eau de la bouteille et la phase organique dans un erlenmeyer de $250 \mathrm{ml}$ contenant 5 à $10 \mathrm{~g}$ de sulfate de sodium anhydre $\left(\mathrm{Na}_{2} \mathrm{SO}_{4}\right)$ pour sécher l'extrait. On répète la même opération une deuxième fois. On laisse l'extrait en contact avec le sulfate de sodium pendant environ $30 \mathrm{~min}$ en agitant de temps en temps et l'on filtre la phase organique sur un tampon de laine de verre disposé au fond de l'entonnoir (préalablement portée à $450{ }^{\circ} \mathrm{C}$ pendant 2 heures) et enfin l'on recueille la phase organique dans un ballon de $250 \mathrm{ml}$.

\section{Evaporation et concentration de l'extrait}

Avant l'analyse, l'extrait organique est évaporé avec l'évaporateur rotatif sous vide (température du bain marie $40{ }^{\circ} \mathrm{C}$, température de l'eau de refroidissement $\left(8-10{ }^{\circ} \mathrm{C}\right)$. Lorsque le volume est réduit à environ $2 \mathrm{ml}$, on transvase dans un récipient gradué de Kuderna Danish et l'on y adjoint quelques $\mathrm{ml} d$ 'hexane provenant du rinçage du ballon.

\section{Purification}

L'évaporation finale est faite par concentration à température ambiante, par un jet azote jusqu'à $2 \mathrm{ml}$. Puis, l'on prélève $400 \mu \mathrm{L}$ d'extrait dans un flacon d'injection avec insert et le reste dans un flacon sans insert.

\section{Analyse}

On fait l'analyse de celui avec insert et l'on garde l'autre pour la confirmation.

\section{Conditions chromatographiques}

Les conditions chromatographiques globales sont les suivantes :

Colonne : $25 \mathrm{~m} * 0,20 \mathrm{~mm} * 0,11 \mu \mathrm{m} \mathrm{HP}-5$

Détecteur: Capture d'électrons (ECD)

Injecteur: Directe sur colonne (On Column)

Gaz vecteur: Hydrogène $\left(\mathrm{H}_{2}\right)$

Pression à l'entrée de l'appareil: 20 psi

Pression à la tête de la colonne : 10 psi

Gaz d'appoint ECD: Azote $\left(\mathrm{N}_{2}\right)$

Pression à l'entrée de l'appareil: 44 psi

Température de l'injecteur: $78{ }^{\circ} \mathrm{C}$ (Oven Track

on)

Température du détecteur: $280^{\circ} \mathrm{C}$; température atteinte selon le processus suivant: $75{ }^{\circ} \mathrm{C}$ pendant $1,5 \mathrm{~min} ; 30{ }^{\circ} \mathrm{C} / \mathrm{min}$ à $175{ }^{\circ} \mathrm{C}$; 2,5

${ }^{\circ} \mathrm{C} /$ min à $210{ }^{\circ} \mathrm{C} ; 10{ }^{\circ} \mathrm{C} /$ min à $275{ }^{\circ} \mathrm{C} ; 275^{\circ} \mathrm{C}$ pendant $2 \mathrm{~min}$.

Volume injecté: $1 \mu \mathrm{l}$

Colonne $1 \mathrm{HP}-5$ : $25 \mathrm{~m} * 0.2 \mathrm{~mm} * 0.11 \mu \mathrm{m}$

Colonne 2 HP-50+: $30 \mathrm{~m} * 0.25 \mathrm{~mm} * 0.15 \mu \mathrm{m}$.

\section{Quantification}

Les concentrations des pesticides contenus dans l'échantillon sont calculées automatiquement par le logiciel ChemStation en comparant les surfaces des pics des produits de l'échantillon à celles obtenues avec des solutions étalons de référence du Dr EHRENSTORFER GmbH.

$\mathrm{Au}$ total, vingt (20) pesticides organochlorés ont été recherchés. Parmi ces composés, huit (8) sont des polluants organiques persistants (POPs). Il s'agit du DDT, de l'endosulfan, du HCB, du lindane, de 
l'aldrine, du dieldrine, de l'endrine et de l'heptachlore.

\section{RESULTATS ET DISCUSSION}

Difficultés rencontrées et moyens utilisés

La première difficulté rencontrée a été surtout liée à la mauvaise collaboration des maraîchers pendant les investigations sur le terrain. Ceux-ci étaient méfiants, compte tenu du fait que l'activité est interdite. L'inventaire des pesticides de synthèse utilisés par les exploitants maraîchers est présenté dans le Tableau 3.

Les photos 1 à 6 montrent les différentes activités entreprises aux abords du barrage. Comme autres activités, les populations viennent prélever de l'eau du barrage comme eau de boisson car elles sont loin des quelques forages autour du barrage, mais aussi pour les activités économiques telle que la préparation du dolo (bière locale). Elles effectuent parfois la lessive aux abords immédiats du cours d'eau. De même, les animaux viennent s'abreuver directement dans le barrage et leurs déjections (photo 5) couvrent les alentours de celui-ci.

La deuxième difficulté est la rareté de la documentation relative au thème de l'étude.

La troisième difficulté est due au fait qu'au laboratoire, nous n'avons pas pu effectuer toutes les analyses prévues à cause du manque de matériel et de réactifs. Ainsi donc, seuls les pesticides organochlorés ont été recherchés.

\section{Interprétation des résultats}

Les courbes des Figures 3 à 9 indiquent l'évolution des différents paramètres en fonction du temps et du lieu de prélèvement des échantillons. Nous interpréterons ces résultats paramètre par paramètre.

\section{Le $p H$}

Nous constatons que les valeurs du $\mathrm{pH}$ (Figure 3) sont voisines de la neutralité (7,08 à 8,09 ), valeurs conformes aux normes (MEE décret 185, 2001).

\section{La conductivité}

La conductivité est une mesure de la capacité de l'eau à conduire un courant électrique, donc une mesure indirecte de la teneur de l'eau en ions. Ainsi, plus l'eau contient des ions comme le calcium $\left(\mathrm{Ca}^{2^{+}}\right)$, le magnésium $\left(\mathrm{Mg}^{2+}\right)$, le sodium $\left(\mathrm{Na}^{+}\right)$, le potassium $\left(\mathrm{K}^{+}\right)$, les ions bicarbonates $\left(\mathrm{HCO}_{3}{ }^{-}\right)$, les ions sulfates $\left(\mathrm{SO}_{4}{ }^{2-}\right)$ et les ions chlorures $\left(\mathrm{Cl}^{-}\right)$, plus elle est capable de conduire un courant électrique et plus la conductivité mesurée est élevée.

Les caractéristiques des eaux de forages et de puits selon la conductivité (Hade, 2002) sont indiquées dans le Tableau 4.

La Figure 4 contient les valeurs de la conductivité enregistrées dans notre étude. Elles sont situées pour la plupart entre 60 et $77 \mu \mathrm{S} / \mathrm{cm}$. Ces valeurs sont assez faibles, comparées aux valeurs de la conductivité en eau douce (moins de $200 \mu \mathrm{S} / \mathrm{cm}$ ) (Hade, 2002), ce qui correspond à une minéralisation peu importante pour les eaux du barrage de Ziga, même pour le point $\mathrm{S} 1$ qui est situé en aval du barrage où la conductivité a une valeur plus élevée que celle des autres points. Pendant la saison sèche, l'aval est alimenté par des forages.

\section{La turbidité}

Les valeurs de la turbidité sont comprises entre 182 et 211 NTU (Figure 5). Cela s'explique par l'absence de couverture végétale près du barrage. La turbidité est due aux matières en suspension (MES) aux colloïdes (électrolytes dissous : les argiles et limons). La turbidité et la formation d'algues sont inversement proportionnelles (De Villiers et al., 2005). 


\section{Les matières en suspension (MES)}

Nous remarquons qu'au mois de mars, la turbidité et les MES ont légèrement baissé (Figure 6). Cela peut s'expliquer par le phénomène de décantation naturelle en l'absence de perturbation due aux eaux de ruissellement. L'accroissement de la turbidité a un impact sur le processus biologique du fait d'une modification de la température. Le blocage du passage de la lumière par les sédiments en suspension a un effet sur le phénomène de photosynthèse. La stabilité d'une suspension colloïdale résulte de l'équilibre entre les interactions attractives et les interactions répulsives qui s'exercent sur les particules. Ces interactions dépendent notamment de la température, du $\mathrm{pH}$ et des électrolytes: les argiles et les limons forment des suspensions colloïdales qui floculent en présence de métaux ou de sels.

\section{Les nitrates}

En cas de pluie, les ions nitrates non assimilés par les plantes sont entraînés par lessivage vers les barrages. L'apport de nitrates dans ce barrage entraîne une augmentation des plantes et algues en surface et peut aboutir à une eutrophisation du milieu. Sa présence excessive peut aussi provoquer un problème de santé publique important tel que la méthémoglobinémie. Bien que les valeurs obtenues des ions nitrates (entre 0,2 et 3,4 $\mathrm{mg} / \mathrm{L}$ ) soient conformes aux normes nationales, nous remarquons leur augmentation au mois de mars (Figure 7).

\section{Le phosphore}

Le phosphore présent dans l'eau (Figure 8) est dû à l'épandage d'engrais dans les champs et dans les cultures maraichères sur les berges du barrage. Cette présence pourrait être expliquée par le fait que les phosphates des engrais se fixent sur le sol et sont entraînés par les eaux de ruissellement. Le phosphore est transporté en solution dans les retenues d'eau et se fixe aux sédiments qui le libèrent par la suite.

\section{La chlorophylle « $a$ »}

Pour caractériser au mieux l'état trophique des lacs, on évalue le degré d'eutrophisation mesurant les concentrations en phosphore total en chlorophylle-a et en déterminant la clarté des eaux à l'aide du disque de Secchi. En quantifiant ces paramètres pour caractériser la qualité de l'eau, on peut établir une classification des niveaux d'eutrophisation des plans d'eau (Devidal et al., 2007). Dans le cas de cette étude, la caractérisation a été faite en dosant la teneur en chlorophylle «a » (Figure 9).

A travers le Tableau 5, on peut dire que les eaux du barrage sont ultra oligotrophes, la teneur en chlorophylle «a » étant largement inférieure à $1 \mu \mathrm{g} / \mathrm{L}$.

\section{Analyse des pesticides}

Le Tableau 6 nous donne les résultats de nos analyses sur les pesticides.

Sur les vingt (20) composés recherchés, cinq (5) ont été retrouvés. Il s'agit de l'aldrine, de l'endosulfan sulfate, du alpha endosulfan, du beta $\mathrm{HCH}$ et du o,p DDE. L'endosulfan sulfate a été retrouvé à l'état de traces dans les échantillons E'2 et S'2, l'aldrin à une concentration de $0,056 \mu \mathrm{g} / \mathrm{L}$ dans l'échantillon W'2, l'alpha endosulfan à une concentration de $0,68 \mu \mathrm{g} / \mathrm{L}$ dans l'échantillon W'2, le o,p DDE à l'état de traces dans l'échantillon W'1 et à une teneur de 0,028 $\mu \mathrm{g} / \mathrm{L}$ dans l'échantillon E'7. Enfin, le beta$\mathrm{HCH}$ a été retrouvé à une concentration de $0,19 \mu \mathrm{g} / \mathrm{L}$ dans l'échantillon W'3 (Figure 10) et à $0,019 \mu \mathrm{g} / \mathrm{L}$ dans l'échantillon S'1 (Figure 11). Toutes ces concentrations sont inférieures aux normes admises (CEE, 1975). 


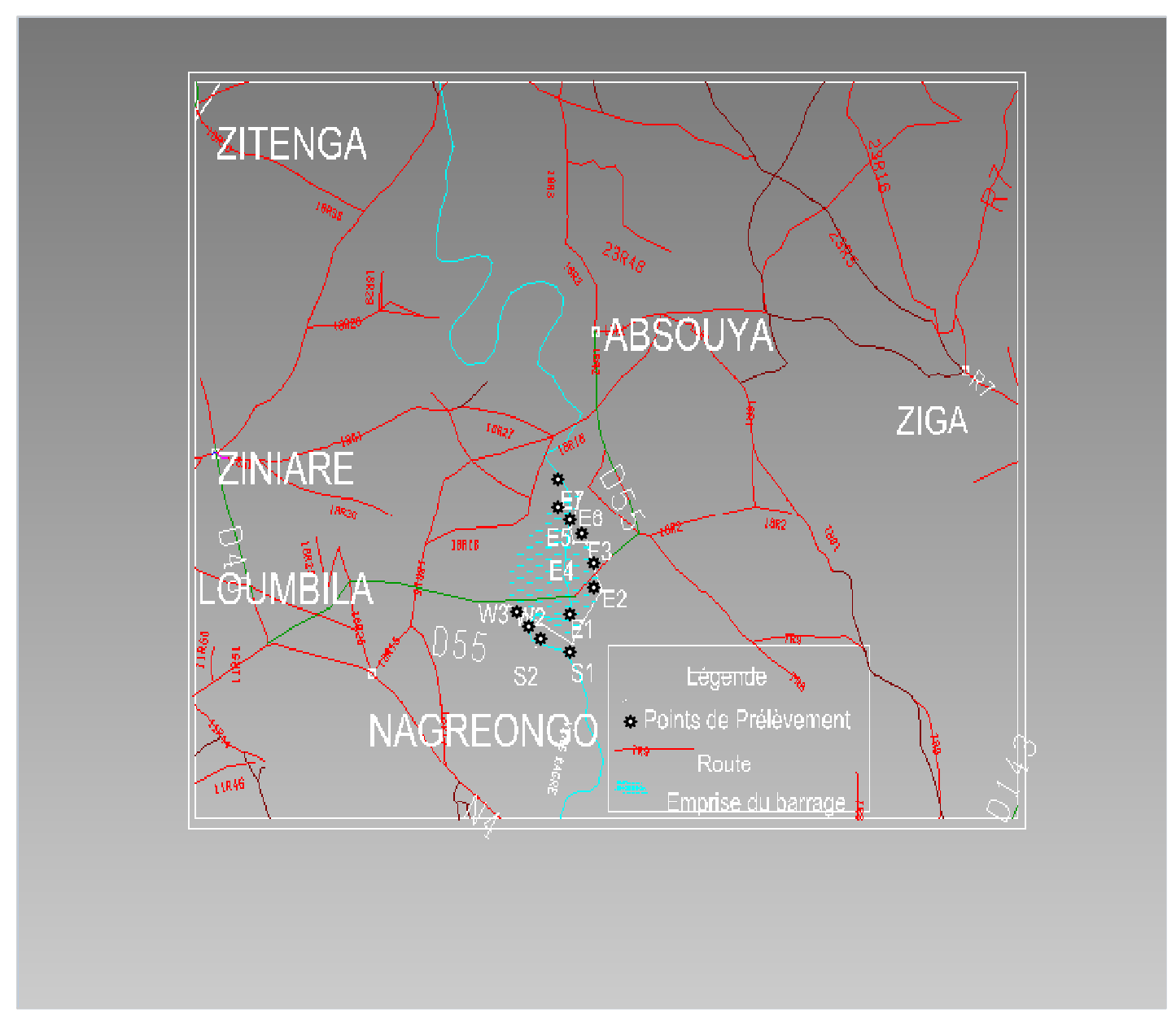

Figure 2 : Cartographie des points de prélèvement.

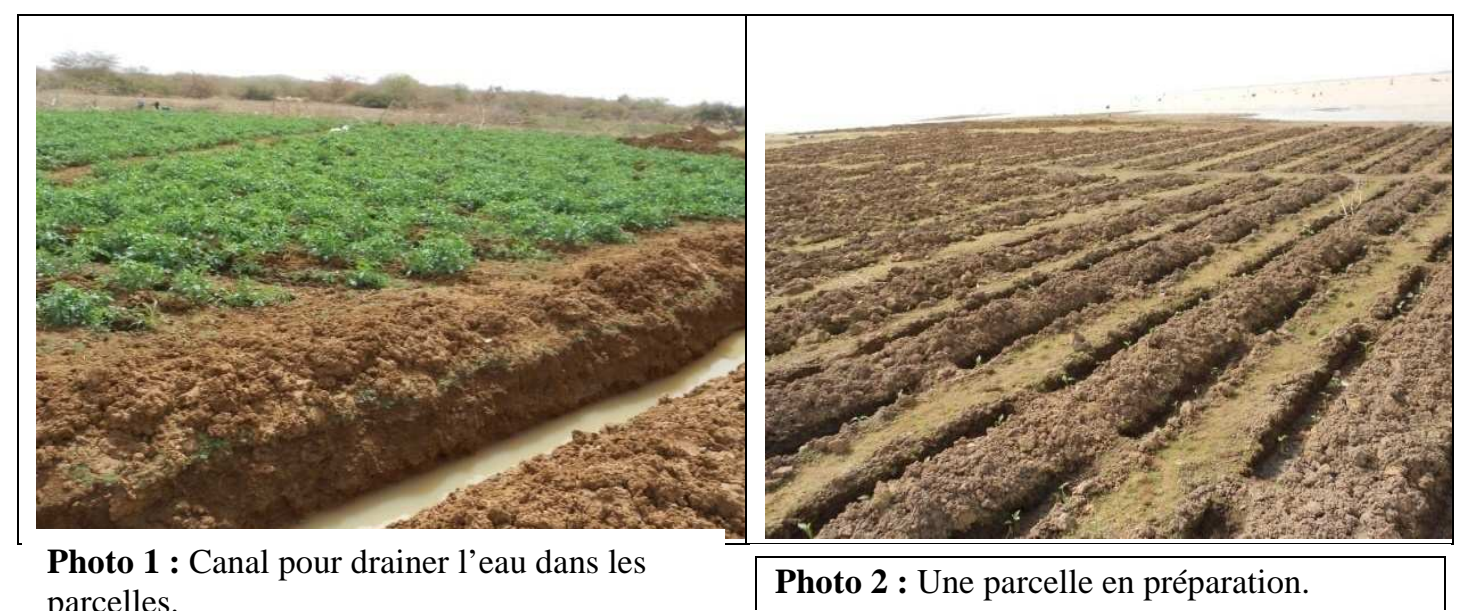




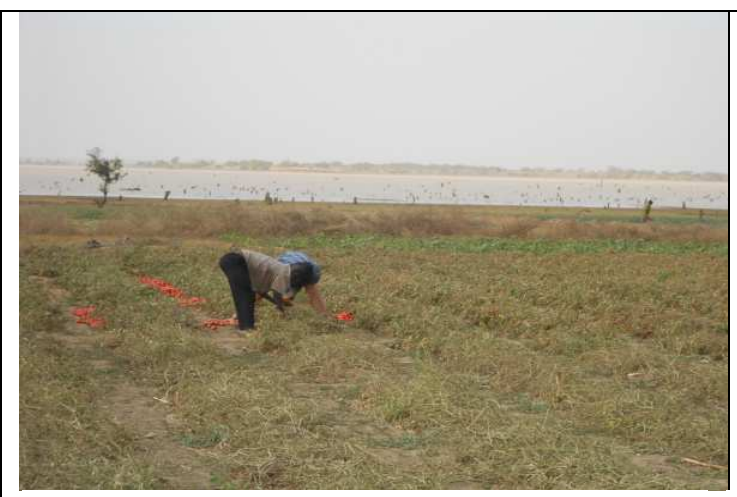

Photo 3 : Champ de tomate.

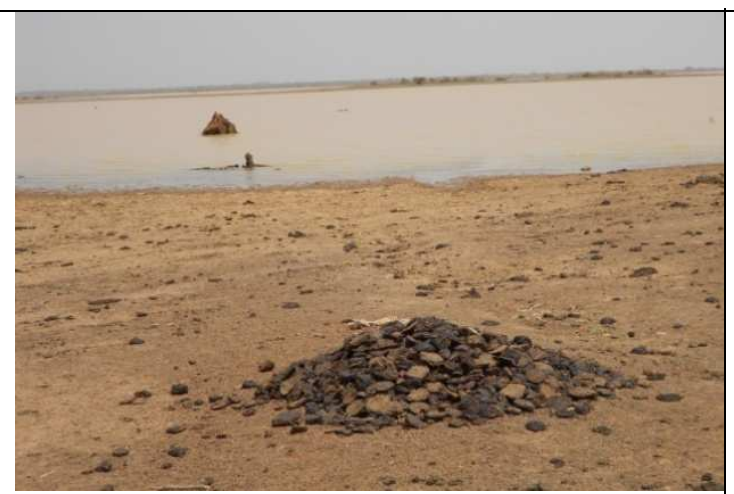

Photo 5 : Un tas de déjections animales.

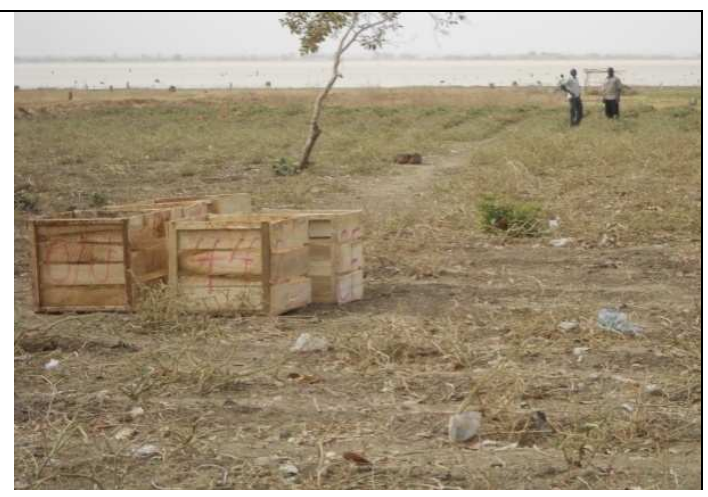

Photo 4 : Conteneurs pour acheminer la tomate.

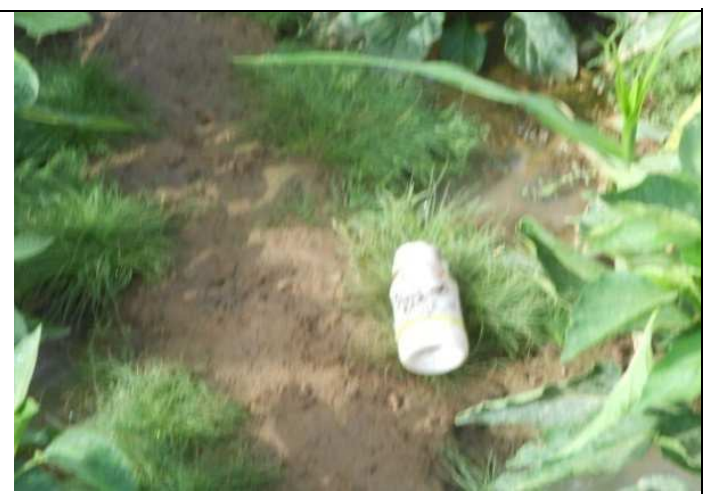

Photo 6 : Boite de pesticide dans un jardin.

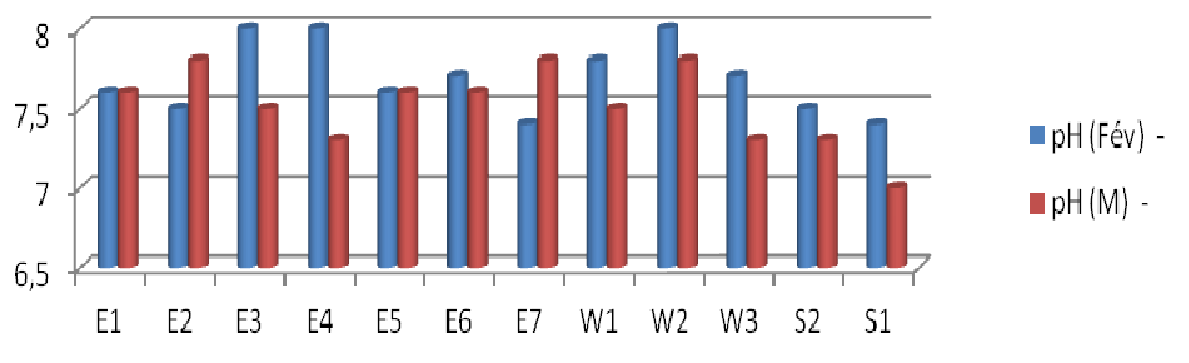

Figure 3: Evolution du $\mathrm{pH}$ en fonction du temps et du lieu de prélèvement (les sigles Fév et $\mathrm{M}$ signifient respectivement février et mars). 


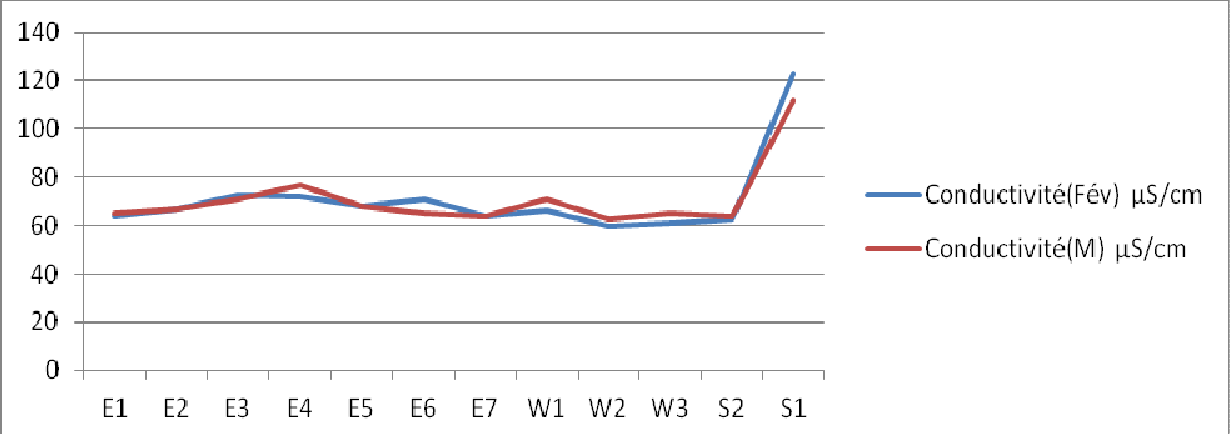

Figure 4: Evolution de la conductivité en fonction du temps et du lieu de prélèvement (les sigles Fév et M signifient respectivement février et mars).

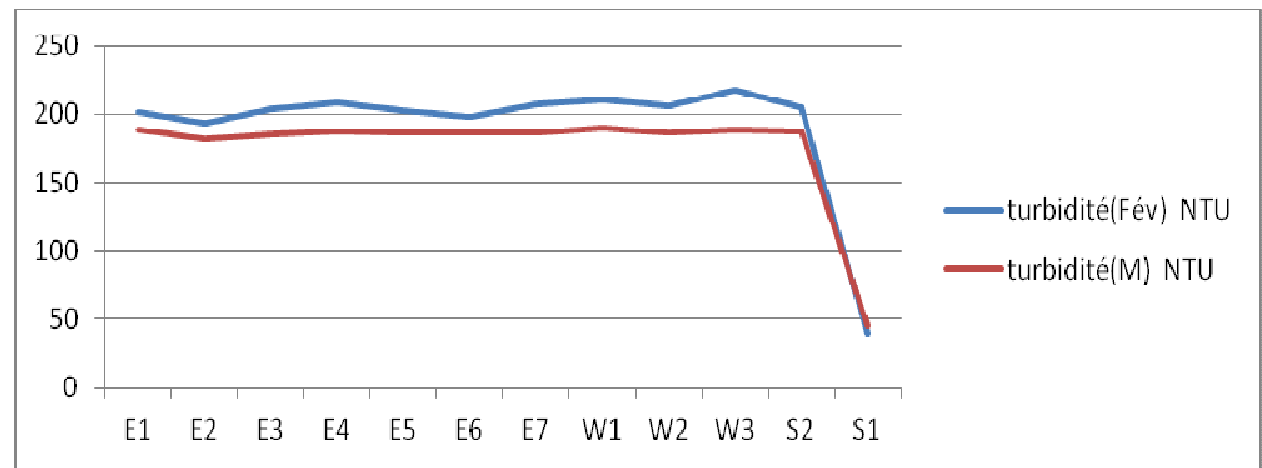

Figure 5: Evolution de la turbidité en fonction du temps et du lieu de prélèvement (les sigles Fév et M signifient respectivement février et mars).

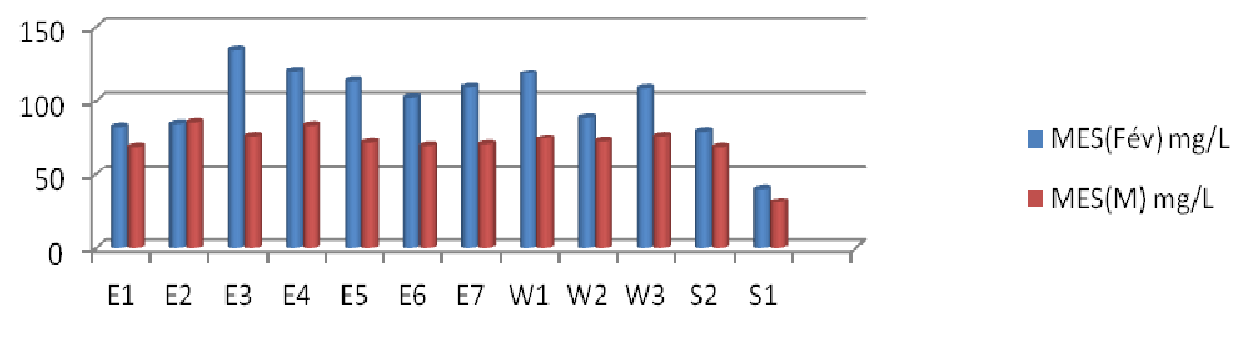

Figure 6: Evolution des MES en fonction du temps et du lieu de prélèvement (les sigles Fév et M signifient respectivement février et mars). 


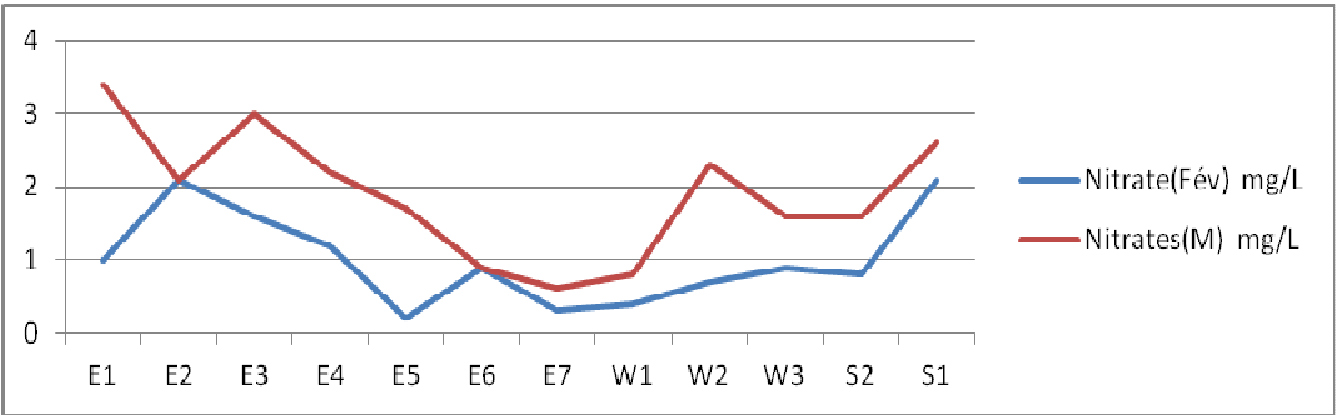

Figure 7: Evolution des nitrates en fonction du temps et du lieu de prélèvement (les sigles Fév et M signifient respectivement février et mars).

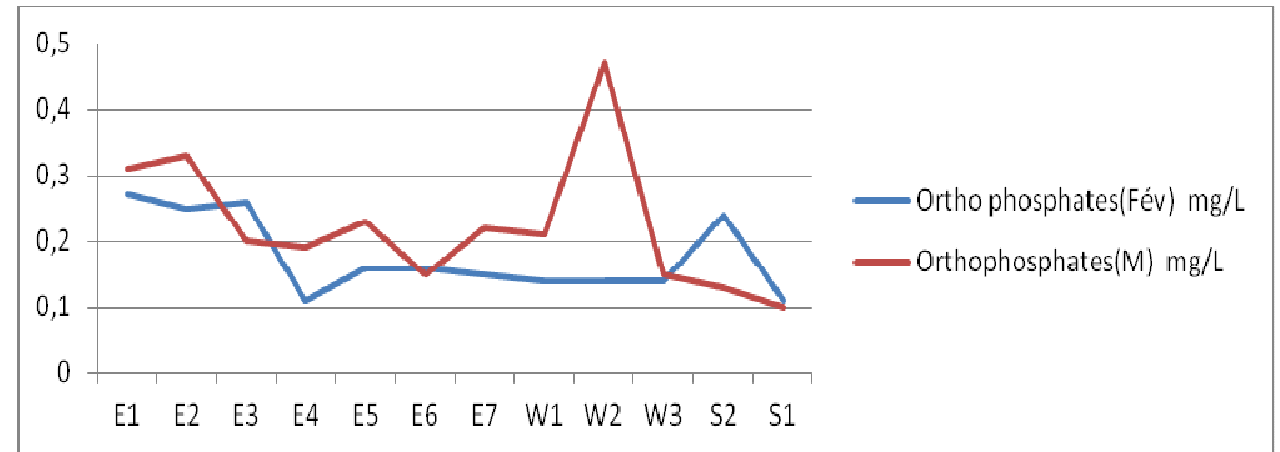

Figure 8: Evolution des ortophosphates en fonction du temps et du lieu de prélèvement (les sigles Fév et M signifient respectivement février et mars).

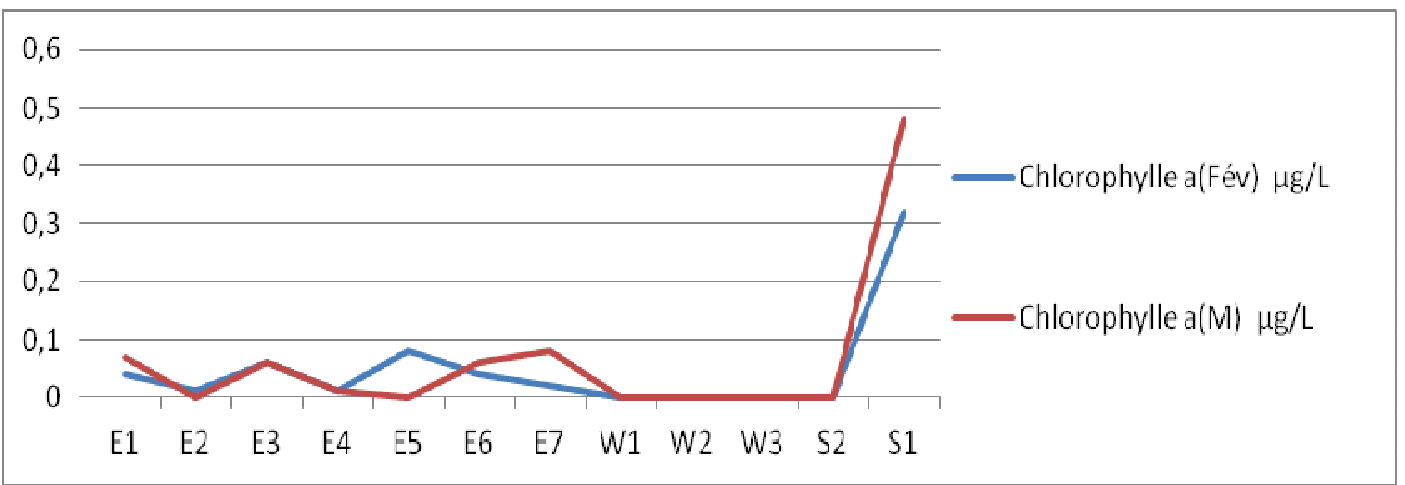

Figure 9: Evolution de la chlorophylle en fonction du temps et du lieu de prélèvement (les sigles Fév et M signifient respectivement février et mars). 
Additional Info: Peak(s) manually integrated

ECD1 B, Back Signal (PEST280312KIDEFAULTB.D)

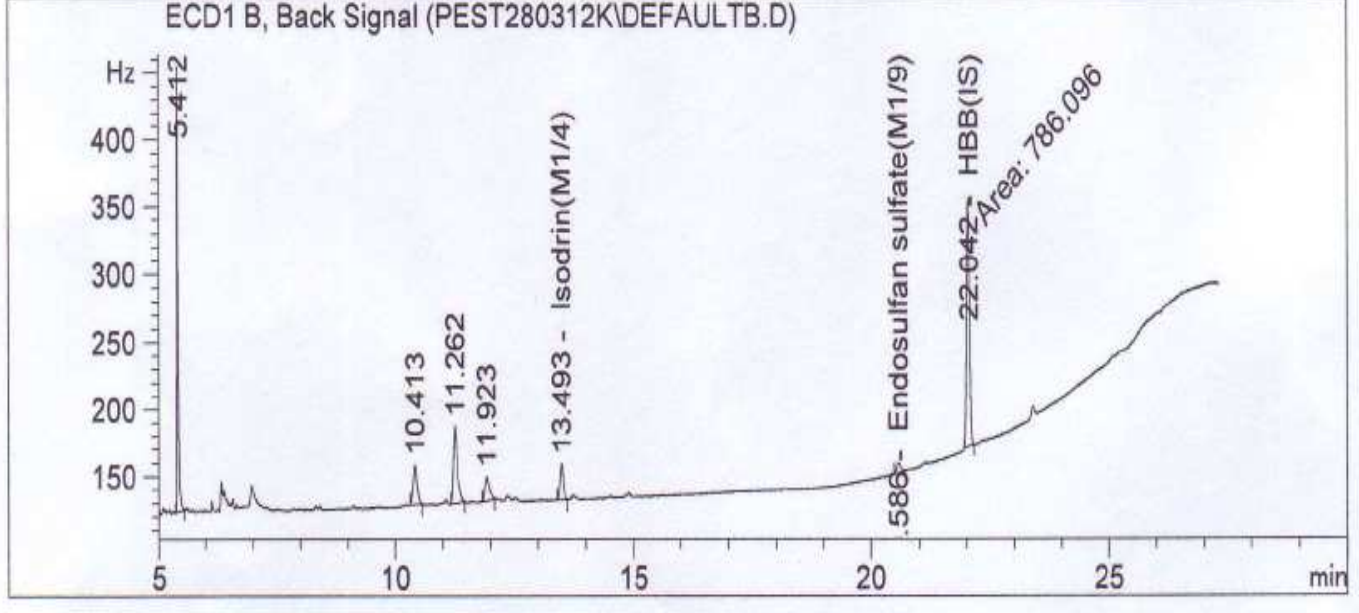

Figure 10: Chromatogramme de l'échantillon W'3 sur CPG.

\section{Additional Info : Peak(s) manually integrated}

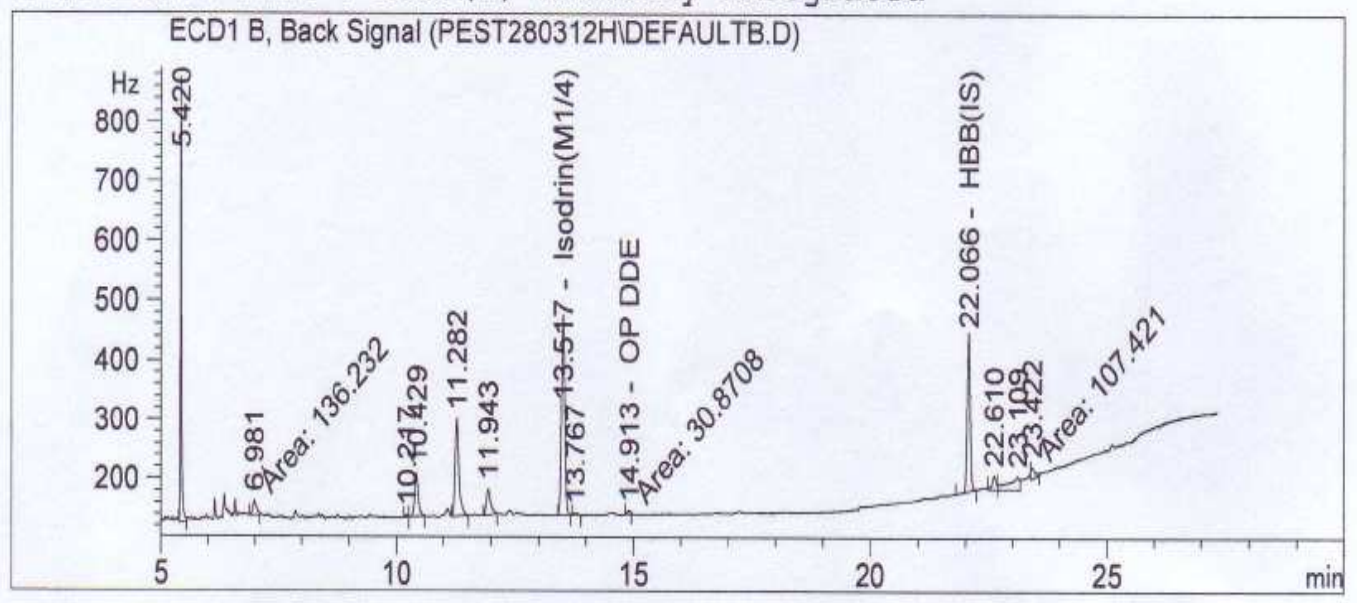

Figure 11: Chromatogramme de l'échantillon W'1 sur CPG. 
Tableau 1 : Levées GPS des points de prélèvement.

\begin{tabular}{|c|c|}
\hline Code d'identification & Localisation \\
\hline & 30P 079860 \\
\hline \multirow[t]{2}{*}{ E1 } & UTM 1382229 \\
\hline & 30P 0711164 \\
\hline \multirow[t]{2}{*}{ E2 } & UTM 1383945 \\
\hline & 30P 0710151 \\
\hline \multirow[t]{2}{*}{ E3 } & UTM 1386496 \\
\hline & 30P 0709239 \\
\hline \multirow[t]{2}{*}{ E4 } & UTM 1386496 \\
\hline & 30P 0709053 \\
\hline \multirow[t]{2}{*}{ E5 } & UTM 1387337 \\
\hline & 30P 0709479 \\
\hline \multirow[t]{2}{*}{ E6 } & UTM 1388439 \\
\hline & 30P 0709578 \\
\hline \multirow[t]{2}{*}{ E7 } & UTM 1389232 \\
\hline & $30 \mathrm{P} 0228333$ \\
\hline \multirow[t]{2}{*}{ W1 } & UTM 1383109 \\
\hline & 30P 0705479 \\
\hline \multirow[t]{2}{*}{$\mathrm{W} 2$} & UTM 1382969 \\
\hline & 30P 0704830 \\
\hline \multirow[t]{2}{*}{ W3 } & UTM 1383109 \\
\hline & 30P 0706729 \\
\hline \multirow[t]{2}{*}{$\mathrm{S} 1$} & UTM 1380892 \\
\hline & 30P 0706729 \\
\hline $\mathrm{S} 2$ & UTM 1381112 \\
\hline
\end{tabular}

Tableau 2: Méthodes et appareillage utilisés pour l'analyse des paramètres physicochimiques.

\begin{tabular}{llll}
\hline Paramètres & Méthode d'analyse & Appareillage & Référence \\
\hline $\mathrm{pH}$ & Electrochimie & $\begin{array}{l}\mathrm{pH} \text {-mètre HANNA } \\
\text { instruments HI } \\
98230\end{array}$ & $\begin{array}{l}\text { Méthode Conforme à la } \\
\text { norme française NFT 90-008 }\end{array}$ \\
& & Multi paramètre & \\
\hline Température & Incorporée aux sondes & WTW multi 340i & \\
& $\mathrm{pH}$, Conductivité, $\mathrm{O}_{2}$ & WTWéthode conforme à la norme \\
\hline Conductivité & Electrochimie & Multi paramètre & Méthou \\
& & WTW multi 340i & française NF T 90-031 \\
\hline Turbidité & Méthode basée sur la & Turbidimètre & $\begin{array}{l}\text { Méthode conforme à la norme } \\
\text { française NF ISO 7027 }\end{array}$ \\
\hline
\end{tabular}




\begin{tabular}{|c|c|c|c|}
\hline & diffusée & & \\
\hline $\begin{array}{l}\text { Matières en } \\
\text { suspension }\end{array}$ & Spectrophotométrie & $\begin{array}{l}\text { spectrophotomètre } \\
\text { Lange Hach DR } \\
3800\end{array}$ & Méthode photométrique \\
\hline $\begin{array}{l}\text { Ortho } \\
\text { phosphates }\end{array}$ & Spectrophotométrie & $\begin{array}{l}\text { spectrophotomètre } \\
\text { Lange Hach DR } \\
3800\end{array}$ & $\begin{array}{l}\text { Méthode Conforme à la } \\
\text { norme française NFT 90-023 }\end{array}$ \\
\hline $\begin{array}{l}\text { Nitrates, } \\
\text { Nitrites }\end{array}$ & Spectrophotométrie & $\begin{array}{l}\text { spectrophotomètre } \\
\text { Lange Hach DR } \\
3800\end{array}$ & $\begin{array}{l}\text { Méthode } 8507 \text { du manuel } \\
\text { d'utilisation du } \\
\text { spectrophotomètre DR } 3800\end{array}$ \\
\hline $\begin{array}{l}\text { Chlorophylle } \\
\text { «a» }\end{array}$ & Spectrophotométrie & $\begin{array}{l}\text { Spectrophotomètre } \\
\text { UV-VIS Shimadzu } \\
\text { UV-160 } 1\end{array}$ & $\begin{array}{l}\text { Méthode ne fait pas } \\
\text { actuellement l'objet d'une } \\
\text { norme (NFT } 90117 \text { ) }\end{array}$ \\
\hline $\begin{array}{l}\text { Pesticides } \\
\text { organochlorés }\end{array}$ & $\begin{array}{l}\text { Chromatographie en } \\
\text { phase gazeuse }\end{array}$ & $\begin{array}{l}\text { Chromatographes } \\
\text { en phase gazeuse } \\
\text { Agilent } 7890 \mathrm{~A} \text {, }\end{array}$ & $\begin{array}{l}\text { Méthode Conforme à la } \\
\text { norme européenne NF EN } \\
\text { ISO } 6468 \text { de février } 1997 .\end{array}$ \\
\hline
\end{tabular}

Tableau 3 : Liste des pesticides de synthèse utilisés sur les berges du barrage de Ziga.

\begin{tabular}{lll}
\hline Nom de la matière active & Famille chimique & Type de pesticide \\
\hline Carbofuran & Carbamates & Insecticide \\
Lambda cyhalotrine & Pyréthrinoïdes & Insecticide \\
Profénofos & Organophosphorés & Insecticide \\
Cypermethrine & Pyréthrinoïdes & Insecticide \\
endosulfan & organochlorés & Insecticide \\
\hline
\end{tabular}

Tableau 4 : Caractéristiques de l'eau de boisson (Hade, 2002).

\begin{tabular}{ll}
\hline Type d'eau & Conductivité $(\boldsymbol{\mu S} / \mathbf{c m})$ \\
\hline Eau douce & Moins de 200 \\
Eau minéralisée & Entre 200 et 1000 \\
Eau salée & Plus de 2000 \\
\hline
\end{tabular}

Tableau 5: Paramètres permettant l'évaluation du niveau trophique d'un plan d'eau.

\begin{tabular}{lccc}
\hline Degré d'eutrophisation & Phosphore total & $\begin{array}{c}\text { Chlorophylle a } \\
\text { moyenne }\end{array}$ & $\begin{array}{c}\text { Chlorophylle a } \\
\text { maximum }\end{array}$ \\
\hline Ultra oligotrophe & $\mu \mathrm{g} / \mathrm{L}$ & $\mu \mathrm{g} / \mathrm{L}$ & $\mu \mathrm{g} / \mathrm{L}$ \\
Oligotrophe & $<4$ & $<1$ & $<2,5$ \\
Mésotrophe & $<10$ & $<2,5$ & $2,5-8$ \\
Eutrophe & $10-35$ & $2,5-8$ & $8-25$ \\
hypertrophe & $35-100$ & $8-25$ & $25-75$ \\
\hline
\end{tabular}


Y. OUATTARA et al. / Int. J. Biol. Chem. Sci. 6(6): 8034-8050, 2012

Tableau 6 : Résultats d'analyse des pesticides organochlorés sur les échantillons du mois de mars 2012.

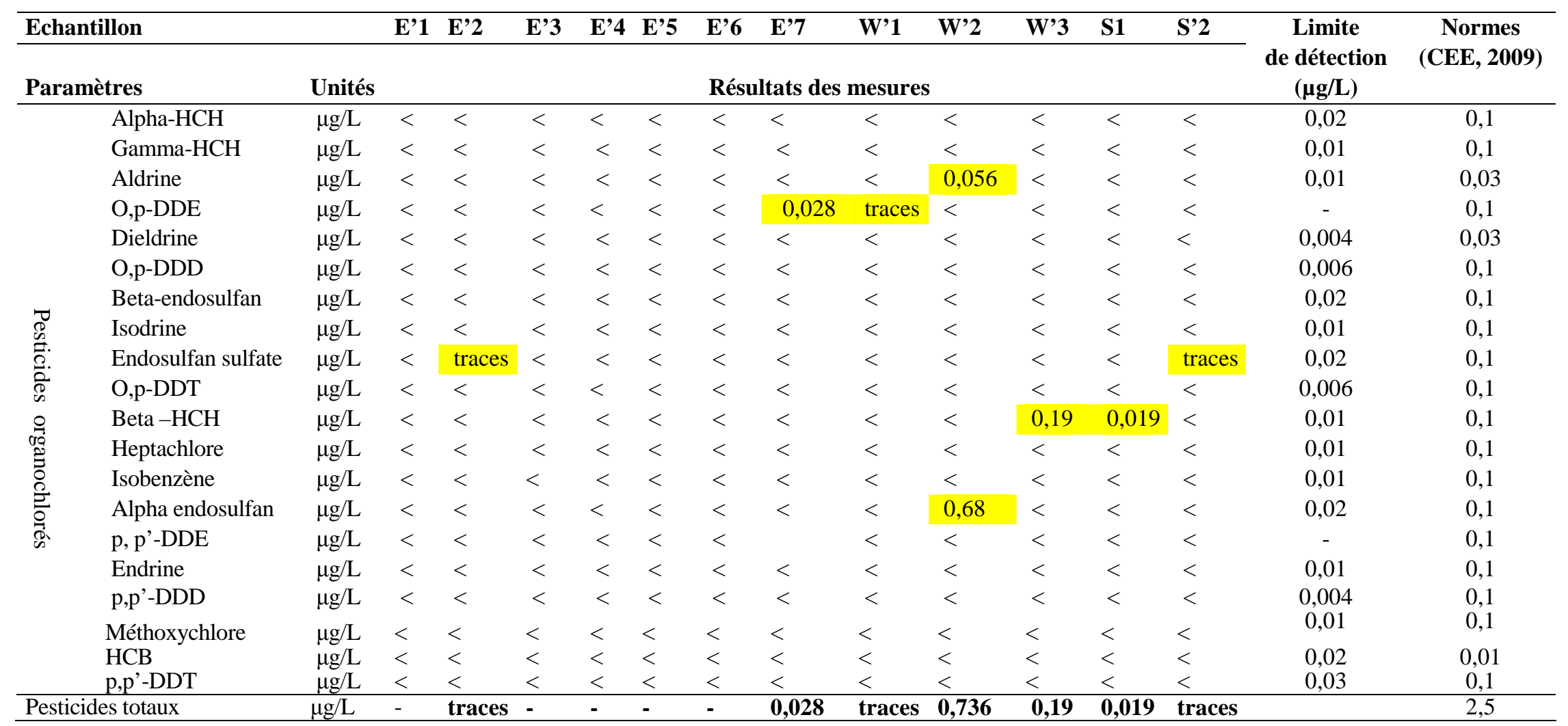

8048 


\section{Conclusion}

Le barrage de Ziga a été construit dans le but d'approvisionner en eau potable la ville de Ouagadougou. Il est situé dans le bassin du fleuve Nakambé, à une quarantaine de km de la ville. Mais des activités maraîchères sont pratiquées dans le lit du barrage par les populations riveraines qui utilisent des pesticides et des engrais susceptibles de polluer l'eau du barrage. L'objectif de notre étude était de déterminer la pollution éventuelle de cette importante réserve d'eau par les divers polluants. Notre étude a consisté à analyser les différents paramètres physicochimiques de l'eau du barrage et à déterminer les concentrations des résidus des pesticides organochlorés utilisés par les maraîchers.

A la date de cette étude, la pollution chimique de l'eau du barrage de Ziga par les pesticides que nous avons pu détecter est faible (CEE, 1975; Liliana, 2007). En effet, aucun pesticide organochloré n'a été détecté à une teneur supérieure à la limite de qualité des eaux brutes du barrage mais des études devraient être menées pour analyser les autres types de pesticides employés par les maraîchers. A cet effet, l'ONEA devrait acquérir des équipements plus performants tels que la CPG/spectromètre de masse pour l'analyse des résidus de toutes sortes de pesticides.

D'autre part, la qualité physicochimique de l'eau étant influencée par le phénomène de l'érosion hydrique lié aux pratiques agricoles et pastorales, il faudrait :

- une volonté politique vigoureuse pour empêcher les activités humaines de se développer sur les berges ;

- la mise en place de périmètres de protection autour des berges ;

- la sensibilisation des maraîchers aux bonnes pratiques agricoles ;

- la pratique des techniques de conservation des sols par les ouvrages tels que les cordons pierreux.
Comme perspectives, au laboratoire, nous n'avons pas pu effectuer toutes les analyses prévues à cause du manque de matériels et de réactifs. Ainsi donc, seuls les pesticides organochlorés ont été recherchés. Dans l'avenir, nous pensons continuer les analyses pendant tout le reste de l'année et surtout étendre cette étude à d'autres familles de pesticides que les organochlorés.

\section{REFERENCES}

Batenbaum JC. 2012. Synthèse de l'Association Santé Environnement sur la pollution de l'eau. Forum Mondial de l'Eau.

Cambon S. 2008. Services d'eau potable, de la logique d'offre à la maîtrise de la demande. Comparaison France-ÉtatsUnis. Thèse en Sciences et Techniques de l'Environnement, Ecole Nationale des Ponts et Chaussées, Paris, 429 pages.

CEE. 1975. Normes des eaux brutes destinées à la production d'eau potable. Extrait de la Directive Européenne du 16 juin 1975 (75/440/CEE).

CEE. 2009. Règlement (CE) n 1107 / 2009 du Parlement Européen et du Conseil du 21 octobre 2009 concernant la mise en place des produits phytopharmaceutiques et abrogeant les Directives 79/117/CEE et 91/414/CEE du Conseil.

Devidal S, Rivard SC, Pouet MF, Thomas O. 2007. Solutions curatives pour la restauration de lacs présentant des signes d'eutrophisation, rapport interne. Observatoire de l'Environnement et du Développement Durable, Université de Sherbrooke, Rappel, Sherbrooke, Québec, Canada, 51 pages.

De Villiers J, Squilbin M, Yourassowsky C. 2005. Qualité physico-chimique et chimique des eaux de surface. Institut Bruxellois pour la Gestion de l'Environnement/Observatoire des Données de l'Environnement, 16 pages.

Djellouli HM, Taleb S. 2005. Qualité chimique et bactériologique des eaux de 
consommation du Sud Algérien. Colloque International sur les Ressources en Eau Souterraines dans le Sahara (CIRESS), Ouargla, Algérie.

Liliana JUC. 2007. Etude des risques liés a l'utilisation des pesticides organochlorés et impact sur l'environnement et la sante humaine. Thèse, Institut de Génie de l'Environnement et Ecodéveloppement, Université Claude Bernard Lyon 1, 227 pages.

Hade A. 2002. Nos Lacs : les Connaître pour Mieux les Protéger. Ministère du Développement durable, de l'Environnement et des Parcs, Réseau de Surveillance Volontaire des Lacs (RSVL). Editions Fides : Canada.

MAHRH. 2001. Etat des lieux des ressources en eau du Burkina Faso et de leur cadre de gestion. Ministère de l'Agriculture, de l'Hydraulique et des Ressources
Halieutiques, version finale, Ouagadougou, Burkina Faso.

MEE décret 185. 2001. Décret $\mathrm{N}^{\circ} 2001$ 185/PRES/PM/MEE du Ministère de l'Environnement et de l'Eau portant fixation des normes de rejet de polluants dans l'air, l'eau et les sols.

Nacro S. 2010. Problématique de l'exploitation à des fins agricoles des berges du barrage de Ziga. Mémoire de fin d'études de DESS, Université de Ouagadougou, Burkina Faso, 49 pages.

OMS/UNICEF. 2007. Atteindre l'OMD relatif à l'eau potable et à l'assainissement, le défi urbain et rural de la décennie.

RGPH. 2006. Ministère de l'Economie et des Finances, Recensement Général de la Population et de l'Habitation RGPH de 2006, 68 pages. 